\title{
Performance Analysis of VolP in WiFi Campus Network
}

\author{
Aiman Ahmed Abu Samra \\ Islamic University of Gaza \\ Gaza, Palestine
}

\begin{abstract}
Voice over Internet Protocol (VoIP) service is growing very fast. Service providers offer VoIP service along with traditional data services using the same IP infrastructure. That is why today it is one of the most dominant technologies for communication. In this paper, simulative investigations have been done for VoIP service in WiFi campus network. Step by step, increasing the number of calls, investigations have been done in terms of important Quality of Service parameters like jitter, packet end-to-end delay, wireless LAN load and wireless LAN throughput.
\end{abstract}

\section{General Terms}

Computer networks, wireless LANs

\section{Keywords}

VoIP, Jitter, delay, 802.11.

\section{INTRODUCTION}

The low cost of voice calls may explain the high demand for VoIP. In addition, VoIP service, use the same IP infrastructure as traditional data services. Because IEEE 802.11 is the most common used technology in today's wireless networks, considerable attention is now being turned to voice over $802.11 x$ family. The main objective of the work presented in this paper is to evaluate the performance of VoIP over wireless local area network. Many factors determine VoIP service quality, including packet loss, delay, delay variation (jitter), and the design of the network [1]. The greatest technical problem in supporting multimedia services over IP is that real-time traffic must reach its destination within a preset time interval (delay) and with some tolerance of the delay variation (jitter). This is difficult because the original UDP/IP operates on a best-effort basis and permits dropping of packets on the way to a destination [2]. The campus wireless LAN in the male section of the Islamic University of Gaza (IUG) was taken as a case study. This wireless LAN consists of six outdoor Access points (AP). The six APs are connected to a $1 \mathrm{Gbps}$ switch. The simulation model was done using OPNET Modeler [3] [4]. OPNET has gained considerable popularity in academia as it is being offered free of charge to academic institutions. That has given OPNET an edge over DES NS2 in both market place and academia [5]. Step by step, increasing the number of calls, investigations have been done in terms of important Quality of Service parameters like jitter, packet end to end delay and throughput. Many papers studied the performance of VoIP, as mentioned in section 2 but to the best of our knowledge none of them studied VoIP over a wireless LAN consisting of a number of access points. The rest of the paper is organized as follows: Section 2 describes a brief overview of related work. Section 3 discusses VoIP, whereas Section 4 deals with the simulation model. Results are discussed in Section 5, finally, the conclusion is presented.

\section{RELATED WORK}

Some researchers like S.Dhanalakshmi et al. [6] and Ritesh Sadiwala et al. [7] investigate the performance of VoIP over wired networks. Other researchers like Tarik et al. [8] and Malhotra [9] studied the Performance of VoIP traffic in WiMAX using various service classes. Syed Rufai et al. [10] studied VoIP performance over WiMAX and LTE. Adnan Hussein Ali [11] studied the performance of IEEE 802.11g LAN in general, he used only one access point with wired LAN and WAN. The author showed the performance optimization via a series of simulation tests with different parameters such as Data rate, and the physical characteristics. Ali M. Alsahlany [12] investigated the performance of VoIP over wireless LAN and WAN using different codecs. He found that G.729A codec gives a significant result for the performance of VoIP than G.711 codecs and G.723.1 codecs. Zainab Alisa [13] investigated an ad hoc network consists of two independent wireless Workstations (WSs). The author concludes that the delay can be decreased with increasing the transmission rate. Delay at the same transmission rate, OFDM is better than DSSS and FHSS physical layer technology. In addition, IR is better than DSSS and FHSS.

\section{VOICE OVER INTERNET (VOIP)}

Quality of Service parameters used in VOIP include: Jitter, delay, packet loss, load and throughput.

\subsection{Jitter}

Jitter is the variation of delay between the two consecutive packets from the T-stream traffic in the output queue [14]. For non-real-time data communications, delayed packets can be stored for an indefinite amount of time at local buffers. For real-time applications like VoIP service, delayed packets may become useless after a prespecified amount of time. The delay jitter buffers hold these "precocious" and delayed packets in an attempt to neutralize the effects of packet interarrival jitter. This helps maintain the real-timeness of real-time communication over packet-switched networks [2].

\subsection{End-To-End delay}

End-to-end delay is the time interval in which a packets travels from one node to another node. VoIP is very sensitive to delay; thus, it must be controlled and managed. As mentioned previously, it is inefficient to wait for all packets arriving in an organized order; therefore, some packets may be dropped if they don't arrive in time and this can cause short periods of silence in the audio stream and can cause bad VoIP quality. Ideally, the delay constraint for VoIP packets is not above $80 \mathrm{~ms}$ [5].

\subsection{Delay}

Transmission time includes delay due to codec processing as well as propagation delay. ITU-T Recommendation G.114 recommends the following one-way transmission time limits for connections with adequately controlled echo (complying with G.131: 
- 0 to 150 ms: acceptable for most user applications;

- 150 to $400 \mathrm{~ms}$ : acceptable for international connections;

- $400 \mathrm{~ms}$ : unacceptable for general network planning purposes; however, it is recognized that in some exceptional cases this limit will be exceeded [1] [15].

\subsection{Packet Loss}

Packet loss is inevitable in IP networks and occurs for various reasons. For example, it occurs when routers or switch work beyond capacity or queue buffers over flow. VoIP network packet loss, above some threshold rate, introduces audio distortions that cause voice quality to be decreased as the rate of packet loss increases [16].

\section{NETWORK MODEL}

The network model presents a campus wireless LAN. This wireless LAN consists of six outdoor Access Points (APs) connected to a one Gbps switch as shown in Figure 1. The general network topology showed in Figure 2. A number of wireless workstations (WSs) are connected to each AP. WSs are suggested to be at the fixed place.

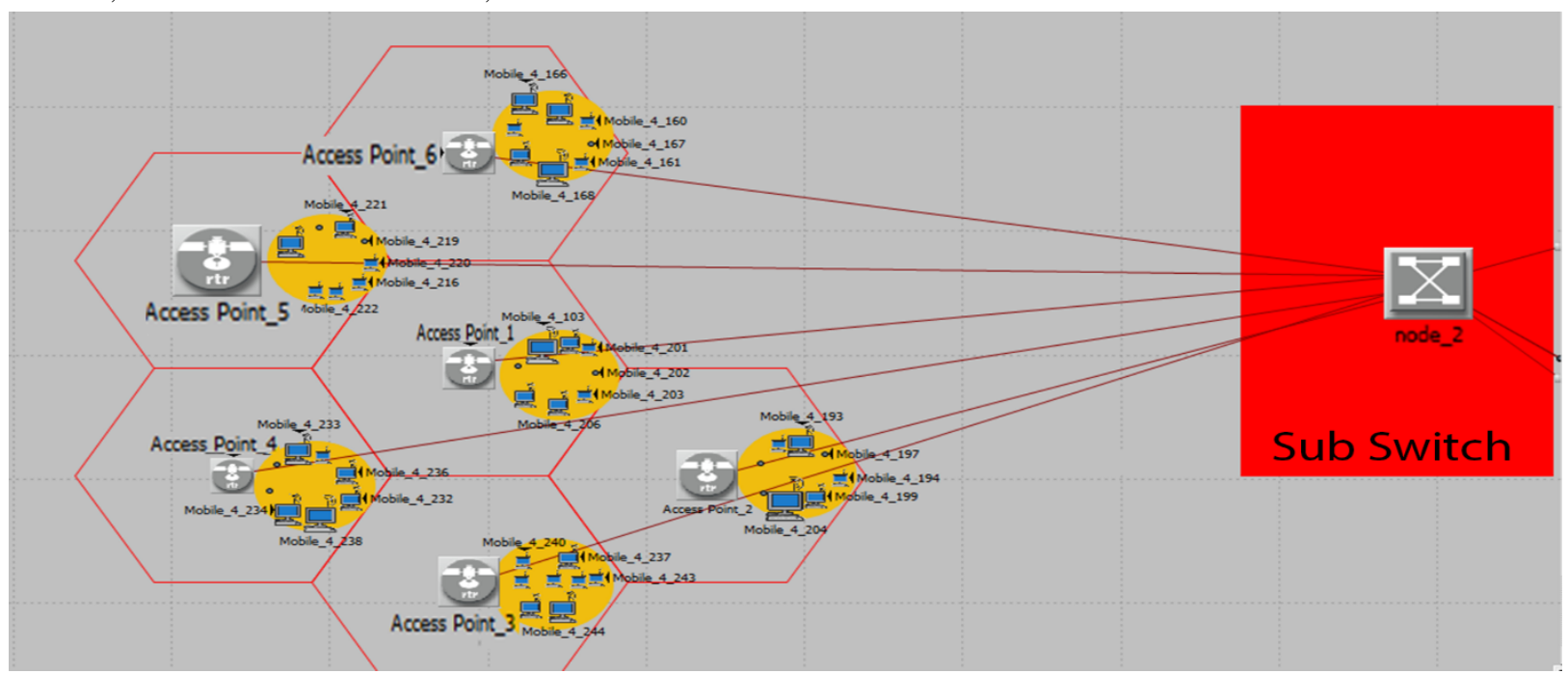

Fig 1: The Network model

Table 1. Voice attributes and their values

\begin{tabular}{|c|c|}
\hline Attribute & Value \\
\hline Silence length (seconds) & default \\
\hline $\begin{array}{c}\text { Talk spurt length } \\
\text { (seconds) }\end{array}$ & default \\
\hline $\begin{array}{c}\text { Symbolic destination } \\
\text { name }\end{array}$ & Voice destination \\
\hline Encoder scheme & G.729A (Silence) \\
\hline Voice frames per packet & none \\
\hline Type of service & Bll discrete \\
\hline RSVP parameters & none \\
\hline Traffic mix & 0.02 \\
\hline Signaling & 0.02 \\
\hline $\begin{array}{c}\text { Compression delay } \\
\text { (seconds) }\end{array}$ & \\
\hline $\begin{array}{c}\text { Decompression delay } \\
\text { (seconds) }\end{array}$ & (a) \\
\hline
\end{tabular}

Step by step, increasing the number of workstations (WSs), four scenarios were implemented, for 4,6,8 and $9 \mathrm{WSs}$ at each AP. Then the values of QoS parameters were taken. To investigate the performance of VoIP with TCP on IEEE 802.11g simulations were performed using OPNET Modeler v14.5. OPNET Modeler is a powerful communication system discrete event simulator (DES) developed by OPNET Technologies. OPNET Modeler 14.5 assists with the design and testing of communications protocols and networks, by simulating network performance for wired and/or wireless environments [4]. The simulation lasts 300 seconds. The attributes that shown in Table 1 were used for the VoIP service. Best effort type of service was used and G.729A (silence) codec as recommended by [12]

\section{RESULTS AND DISCUSSION}

An important parameter in determining the QoS for VoIP is its timing operations. So, the jitter and the delay will be studied initially. The jitter values in all four scenarios are neglected. They are less than 1.3E-11. These values were earned because the network is not congested. Figure 3 shows the average packet end-to-end delay for the four scenarios i.e. the scenarios with $4 \mathrm{WSs}, 6 \mathrm{WSs}, 8 \mathrm{WSs}$ and $9 \mathrm{WSs}$.

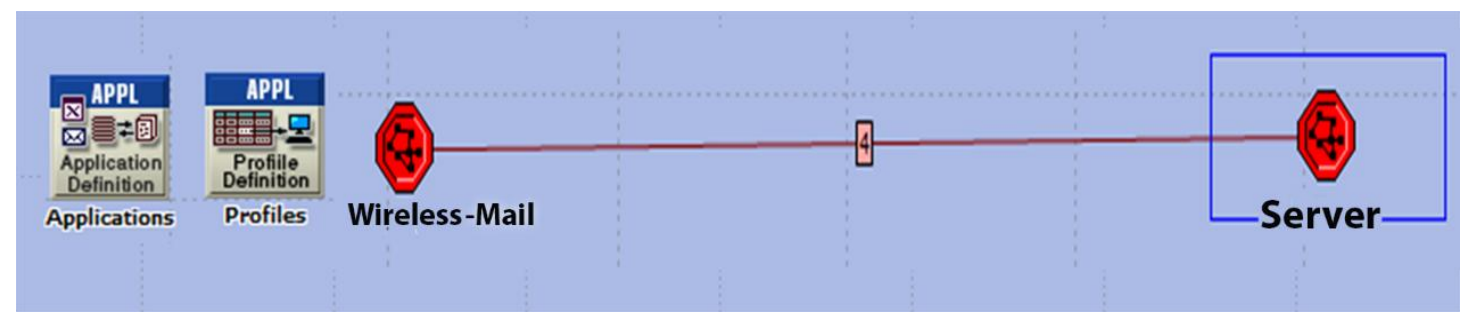

Fig 2: The general network topology 


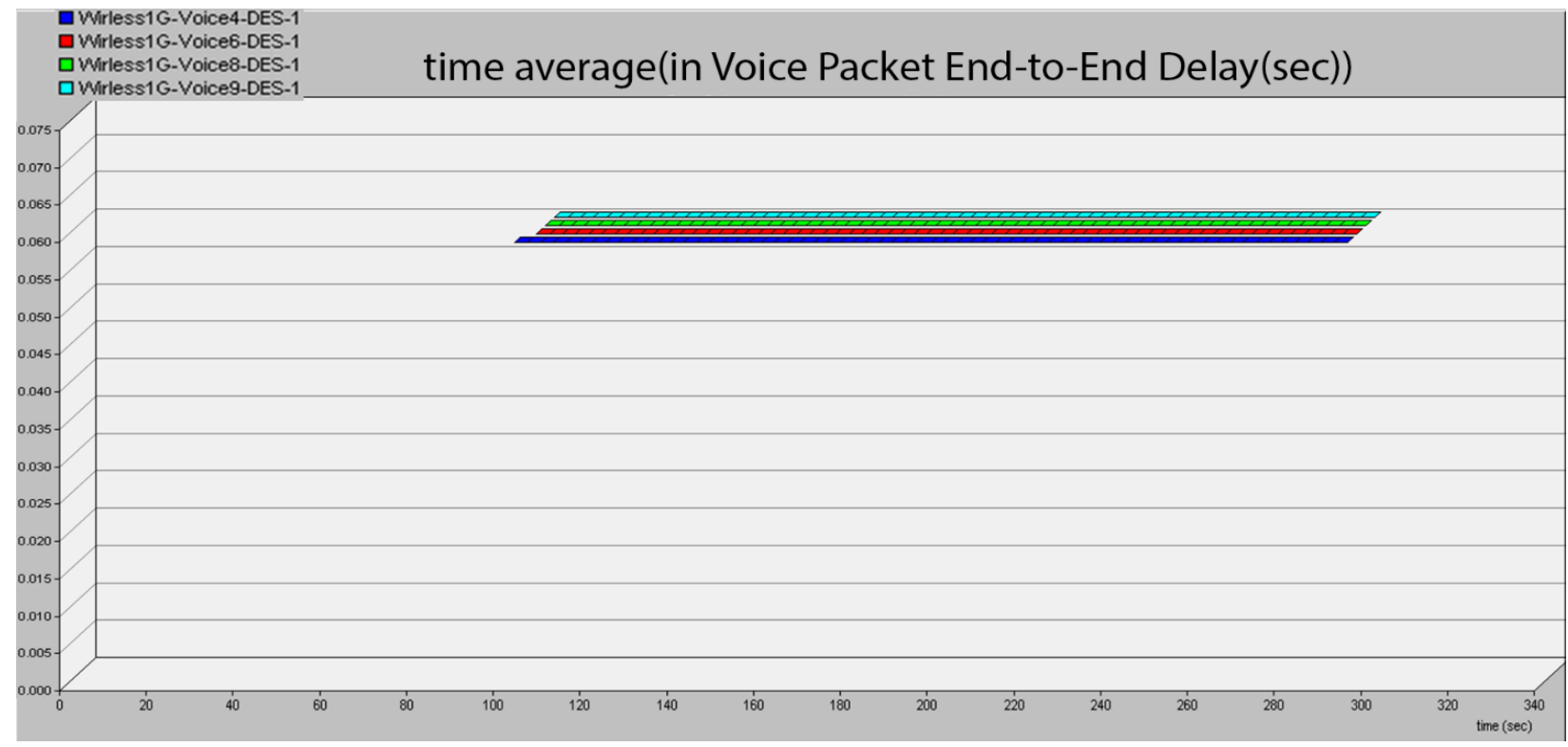

Fig 3: End-To-End delay

The values of end-to-end delay, for all scenarios, are constant because the all scenarios have the same bit rate and the network is not congested.

Wireless LAN delay represents the end-to-end delay of all the packets received by the wireless LAN MACs of all WLAN nodes in the network and forwarded to the higher layer. This delay includes medium access delay at the source MAC, reception of all the fragments individually, and transfer of the frames through AP if access point functionality is enabled [17] [18].

Figure 4 represents the wireless LAN delay. The wireless LAN delay values for the scenarios with 4 WSs, 6 WSs and 8 WSs are very small. They are accepted and even good. But the wireless LAN delay for $9 \mathrm{WSs}$ and $10 \mathrm{WSs}$ reach $270 \mathrm{~ms}$ and $1.2 \mathrm{sec}$ respectively i.e. not accepted. Table 2 presents the values of the wireless LAN delay for the scenarios with 4, 6, 8 and 9 WSs.
Table 2. WLAN delay for the four scenarios

\begin{tabular}{|l|l|l|l|l|}
\hline scenario & $4 \mathrm{WSs}$ & $6 \mathrm{WSs}$ & $8 \mathrm{WSs}$ & $9 \mathrm{WSs}$ \\
\hline Time & $2 \mathrm{~ms}$ & $4 \mathrm{~ms}$ & $26 \mathrm{~ms}$ & $270 \mathrm{~ms}$ \\
\hline
\end{tabular}

The load \& throughput test is concerned with the receipt of the payload data without considering the overhead of network against the load. Load represents the total load (in bits/sec) submitted to wireless LAN layers by all other higher layers in all WLAN nodes of the network. This statistic does not include the bits of the higher-layer packets that are dropped by WLAN MACs upon arrival and not considered for transmission because of, for example, insufficient space left in the higher layer packet buffer of the MAC [17] [19]. The load can be observed in Figure 5.

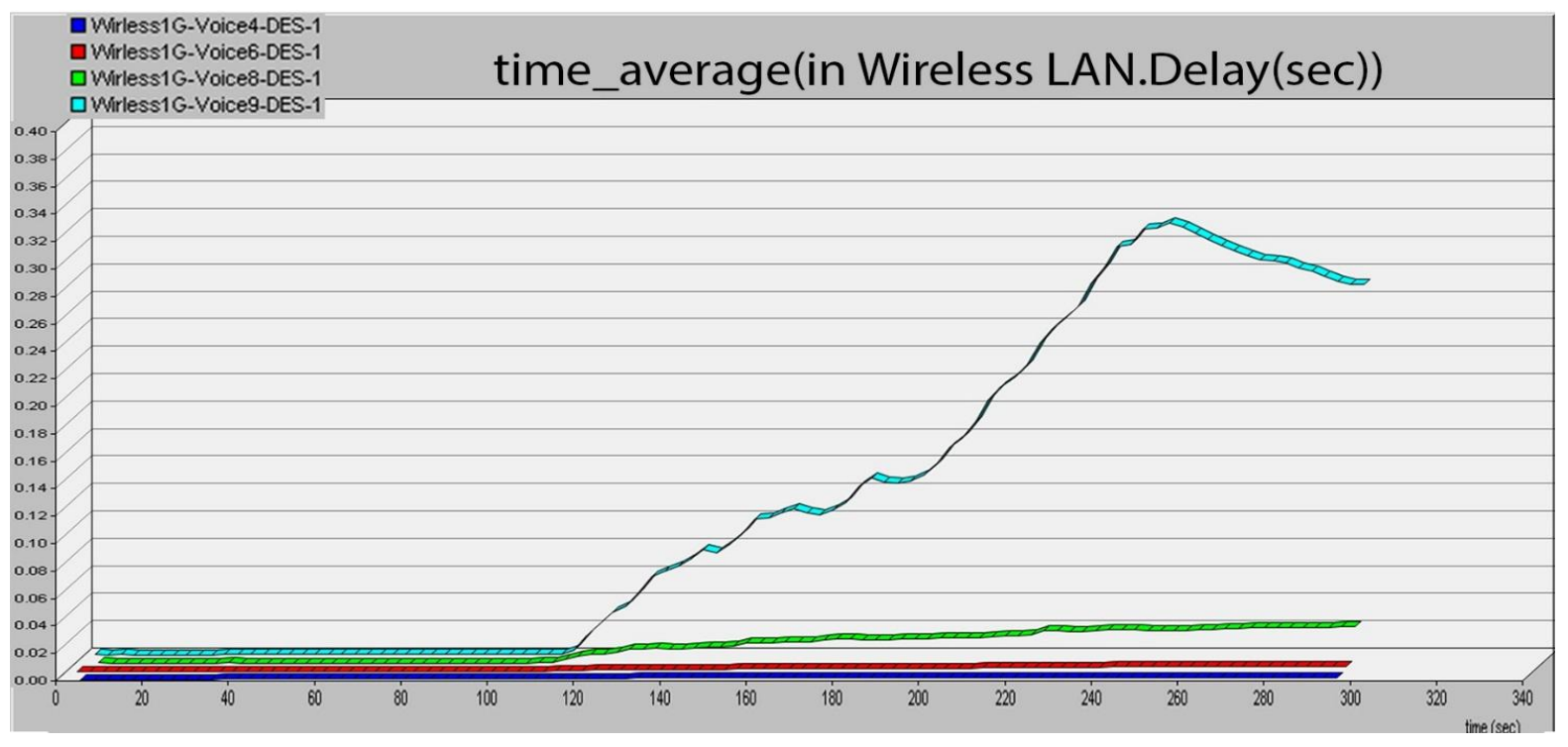

Fig 4: Wireless LAN delay 


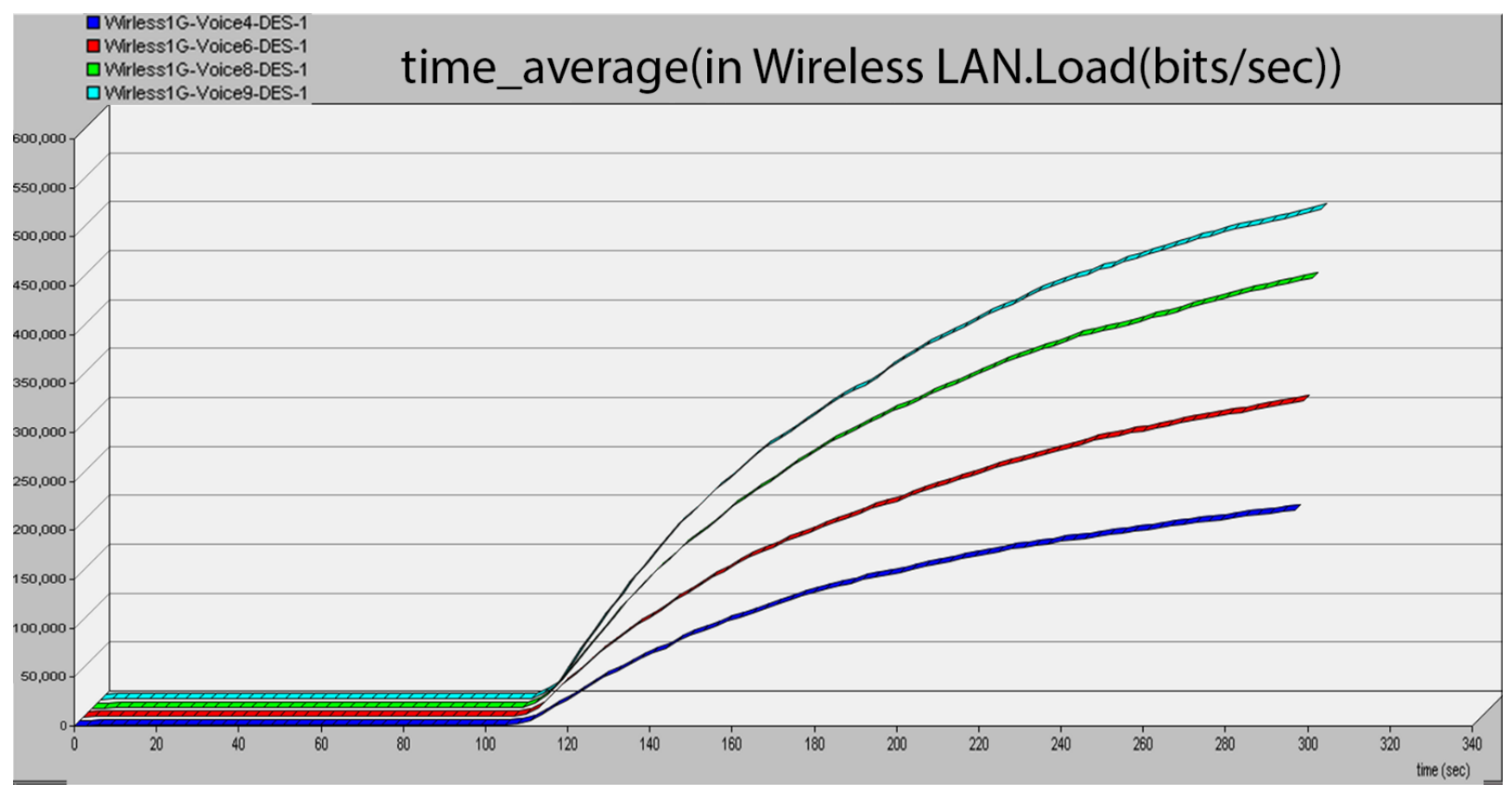

Fig 5: Wireless LAN load

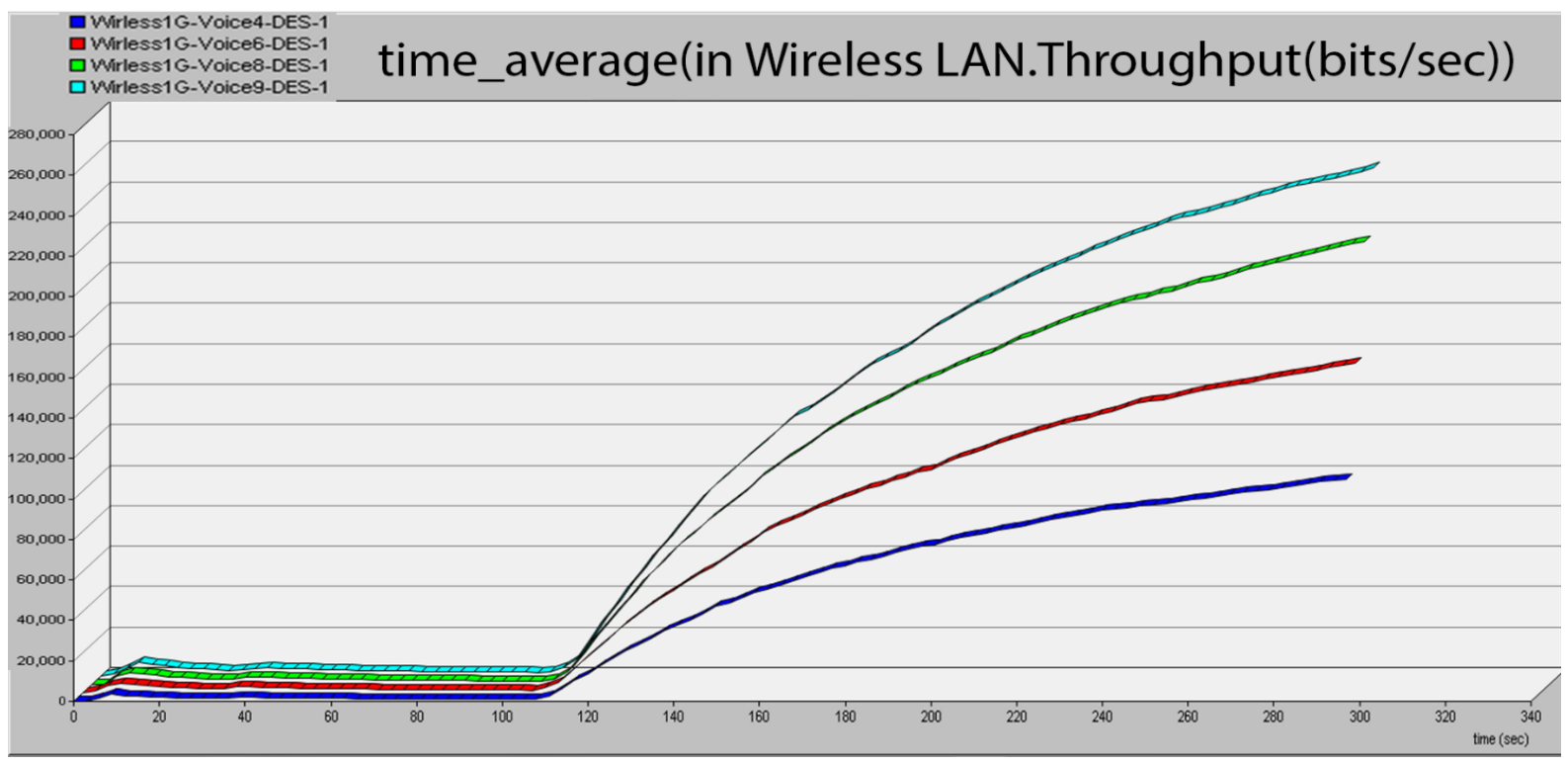

Fig 6: Wireless LAN throughput

Throughput represents the total number of bits (in bits/sec) forwarded from wireless LAN layers to higher layers in all WLAN nodes of the network [17] [19]. Throughput is represented in Figure 6. Increasing the number of the WSs connected to each AP, the Load and the throughput are also increased. But there is no benefit from the increase in throughput for more than $8 \mathrm{WSs}$, because of the increase in the wireless LAN delay, which exceeds the accepted value for VoIP.

From the above figures, it can be concluded that the capacity of the campus wireless LAN in the studied case is 8 WSs using VoIP service for each Access Point. In other words, the capacity of the whole wireless campus LAN is 48 VoIP clients at the same time.

\section{CONCLUSION}

In this paper, simulative investigations have been done for VoIP in WiFi campus network using OPNET Modeler. Step by step, increasing the number of workstations (WSs), investigations have been done in terms of important Quality of Service parameters like jitter, packet end-to-end delay and wireless LAN load and wireless LAN throughput. Based on the simulation results, the jitter values in all scenarios are neglected because the network is not congested. Wireless LAN delay is acceptable for the scenarios with 4,6 , and 8 WSs i.e. the capacity of the campus wireless LAN in the studied case is 8 WSs using VoIP service for each Access Point. The throughput of the wireless networks depends on network load. But there is no benefit from the increase in 
throughput for more than $8 \mathrm{WSs}$, because of the increase in the delay, which exceeds the accepted value for VoIP. As a future work, more types of traffic like Email, FTP and HTTP will be added to make the case more realistic.

\section{REFERENCES}

[1] BUR GOODE, Voice Over Internet Protocol (VoIP), Invited Paper, Proceedings of the IEEE ( Volume: 90, Issue: 9, Sep 2002 ), Page(s): 1495 - 1517

[2] BUR GOODE, IMPLEMENTING VOICE OVER IP, 2003 by John Wiley \& Sons, Inc.

[3] OPNET Technologies, OPNET Modeler Product Documentation Release 11.0, OPNET Modeler, 2005

[4] OPNET https://www.riverbed.com/gb/products/ steelcentral/opnet.html?redirect=opnet

[5] K. Salah and A. Alkhoraidly, An OPNET-based Simulation Approach for Deploying VoIP, International Journal of Network Management Volume 16, Issue 3, Pages 159-183

[6] S.Dhanalakshmi et al, Investigating The Performance Of Voip Over Ethernet Lan In Campus Network. International Journal of Recent Scientific Research Vol. 6, Issue, 6, pp.4389-4394, June 2015

[7] Mr. Ritesh Sadiwala, Dr. Minal Saxena" performance Evaluation of Quality Parameters in VOIP and PSTN Systems" IRACST, Vol.5, No 5, October 2015

[8] Anouari, Tarik, and Abdelkrim Haqiq. "Performance analysis of VoIP traffic in WiMAX using various service classes." arXiv preprint arXiv:1308.0223(2013).

[9] Malhotra, Jyoteesh. "Performance Evaluation of Scheduling Services for VoIP in WiMAX Networks." International Journal of Computer Applications71.19 (2013).

[10] Syed Hamza Mehmood Rufai et al., Comparison of VoIP and Video Content Performance Over WiMAX and LTE, Simon Fraser University, Canada
[11] Adnan Hussein Ali et al., Performance Evaluation of IEEE802.11g WLANsUsing OPNET Modeler, American Journal of Engineering Research (AJER), Volume-02, Issue-12, pp-09-15

[12] Ali M. Alsahlany, studied the PERFORMANCE ANALYSIS OF VOIP TRAFFIC OVER INTEGRATING WIRELESS LAN AND WAN USING DIFFERENT CODECS, International Journal of Wireless \& Mobile Networks (IJWMN) Vol. 6, No. 3 , June 2014

[13] Zainab T. Alisa, Evaluating the Performance of Wireless Network using OPNET Modeler, International Journal of Computer Applications (0975 - 8887) Volume 62No.13, January 2013.

[14] Zheng, Li, Liren Zhang, and Dong Xu. "Characteristics of network delay and delay jitter and its effect on voice over IP (VoIP)." Communications, 2001. ICC 2001. IEEE International Conference on. Vol. 1. IEEE, 2001.

[15] Understanding Delay in Packet Voice Networks https://www.cisco.com/c/en/us/support/docs/voice/voicequality/5125-delay-details.html\#standarfordelaylimits

[16] James, Jim H., Bing Chen, and Laurie Garrison. "Implementing VoIP: a voice transmission performance progress report." IEEE Communications Magazine42.7 (2004): 36-41.

[17] Network Simulation Experiments Manual https://booksite.elsevier.com/9780123850591/Lab_Manu al/Lab_02.pdf 9/8/2017

[18] Bourdoucen, H., A. Al Naamany, and A. Al Kalbani. "Impact of Implementing VPN to Secure Wireless LAN." International Journal of Computer and Information Engineering 3 (2009): 1.

[19] Sari, Arif, and Behnam Rahnama. "Simulation of 802.11 Physical Layer Attacks in MANET." Computational Intelligence, Communication Systems and Networks $(\mathrm{CICSyN}), 2013$ Fifth International Conference on. IEEE, 2013. 